\title{
Mesenchymal Stem Cells During Tumor Formation and Dissemination
}

\author{
Diego Correa ${ }^{1,2}$
}

Published online: 19 April 2016

(C) Springer International Publishing AG 2016

\begin{abstract}
The tumor microenvironment (TME) is composed by malignant and non-malignant cells, all embedded in a dense extracellular matrix (ECM) rich with unstable vessels. Targeting TME components, especially those associated with the vasculature such as endothelial cells (ECs) and pericytes, has shown clinical benefits. The identity correlation between pericytes and mesenchymal stem cells (MSC) has broadened the functional roles of these adult stem cells, now tightly involved in cancer biology. This review summarizes this involvement, focusing on their participation in: 1) skeletal primary malignancies; 2 ) formation of distant primary tumors; 3 ) intravasation of cancer cells at the primary tumors; and 4) extravasation of cancer cells at the target organ. Given their tropism to sites of injury and inflammation, bone marrow (BM)-derived MSC (BM-MSC) follow tumor-derived signals and participate in the formation of distant primary tumors, by repopulating their perivascular habitat and contributing to tumor growth. Thus, targeting primary tumor's pericytes severely reduces growth, yet dissemination of constitutive cancer cells increases. The impact of pericyte-deficient coverage on the target organ is rather opposite, generating a selective reduction of cancer cell invasion in some organs. These roles seem to be founded on the distinct molecular communication
\end{abstract}

This article is part of the Topical Collection on Stem Cells: Policies from the Bench to the Clinic

Diego Correa

dxc821@med.miami.edu

1 Department of Orthopaedics - Division of Sports Medicine, University of Miami, Miller School of Medicine, Miami, FL, USA

2 Diabetes Research Institute \& Cell Transplant Center, University of Miami, Miller School of Medicine, Miami, FL, USA and physical interactions between MSC as pericytes and the cancer cells.

Keywords Mesenchymal Stem Cells (MSC) · Pericytes · Tumor microenvironment $\cdot$ Cancer dissemination . Intravasation $\cdot$ Extravasation

\section{Introduction}

Cancer (primary and metastatic tumors) constitutes the second cause of mortality after heart disease, according to the Centers for Disease Control and Prevention (CDC) (about $22 \%$ in women and $24 \%$ in men) [1]. Metastasis is considered a leading prognostic indicator for patient survival and a major contributor to the mortality associated with cancer, with the skeleton as one of the preferred organs for cancer metastatic dissemination in various primary tumors. Tumors are composed of a tight interplay between malignant and nontransformed cells embedded in a highly dynamic extracellular matrix (ECM) rich in unstable blood vessels, collectively constituting the tumor microenvironment (TME) [2]. The cellular compartment within the TME includes cells from the immune system, ECs, pericytes, adipocytes, MSC and tumorassociated fibroblasts (TAF), some of them arising from the tissue itself, while others are recruited from distant locations, mainly the bone marrow (BM) [3-6].

Current cancer therapies are designed to target not only specific biological functions in cancer cells, but also components of the tumor microenvironment (TME), especially the vasculature (i.e., ECs and pericytes), given its critical role in primary tumor growth and dissemination [7]. Pericytes are recruited close to the endothelium by ECs via PDGF-B signaling, where they attach and help stabilize the blood vessel $[8,9]$. Pericytes constitute a novel therapeutic target based on 
recent evidence showing superior reduction in tumor growth when targeted in parallel with ECs [10]. However, pericyte coverage of the vasculature seems to have opposite effects on growth and metastatic potential of primary tumors [11, 12]. This has led to the appreciation of pericytes as gatekeepers for cancer metastasis [13]. With the increasing evidence of MSC residing in the perivascular space (as pericytes), new concepts regarding MSC involvement in the field of cancer are emerging. These concepts are provided in this review, divided in four main topics and with an emphasis on BM-MSC and on skeletal malignancies: 1) MSC in the BM as the cell of origin in various primary bone neoplasms; 2) The participation of BM-MSC during the formation of distant solid tumors; 3) The role of pericytes at the primary tumor's vasculature during intravasation of cancer cells; and 4) The regulatory role of MSC (as pericytes) at the target organ during cancer cell extravasation.

\section{1) MSC as pericytes}

Pericytes are typically defined as heavily branched cells embedded within the basement membrane of non-muscular microvessels, capillaries and postcapillary venules, and in close contact with the underlying endothelial cells. Initially identified by Rouget in 1863 as adventitial cells, pericytes have received a number of names, including mural cells, perivascular cells, periendothelial cells and deep cells [14]. During development-related vasculogenesis pericytes originate from MSC and neural crests, depending on the specific location [15]. In post-developmental stages, the origin of pericytes is far less clear, with tissue resident stem cells [16] and/or myofibroblasts [17], as well as peripheral pluripotent stem cells derived from the BM $[16,18]$ as potential progenitors. Pericytes have a broad spectrum of functions, including the regulation of the vessel's stability, permeability and tone, as well as the shift between quiescent and angiogenic stages of blood vessels, thus participating in the maintenance of the tissue homeostasis. In addition, pericytes participate in immunological defense reactions (e.g., macrophage-like properties), and help in the synthesis of the basement membrane [14, 19].

The description of the physical location of MSC in vivo has captured the attention of researchers for a long time. Initial studies in the early 1990 s demonstrated that pericytes constitute the cell source for different mesenchymal tissues. For instance, Diaz-Flores et al. described venule pericytes in grafted perichondrium and periosteum as the drivers for neochondrogenesis in muscle [20] and as the source of osteoblasts in periosteal osteogenesis [21], respectively. In parallel, Brighton and colleagues proposed the pericyte as the possible progenitor of osteoblasts, based on in vitro studies in which phenotypic similarities, including matrix deposition and osteogenic markers, were established between them [22, 23]. This direct functional relationship between mesenchymal progenitors (i.e., MSC) and pericytes was then confirmed with the detailed description of the mesenchymal stem cell niche, present in almost all post-natal organs and tissues, and in which both cell types share identity and functions [24-32]. Nevertheless, the cellular, molecular and physical complexity of the stem cell niche, along with descriptions of restricted differentiation by both MSC and pericytes, have suggested that the niche is populated by heterogeneous cell types and/ or that not all pericytes are MSC [33].

Finally, this phenotypic/functional relationship between MSC and pericytes has also been evident with malignancies derived from the perivascular niche. Given their established mesenchymal potential, they have been associated with multiple soft tissues and bone-specific malignancies of mesenchymal origin [14], as well as with vascular primary tumor, such as the myopericytoma that exhibits a myoid differentiation pattern [34].

\section{2) MSC in primary bone tumors}

The most prevalent primary tumors in bone are Osteosarcoma (OS), Chondrosarcoma (CS) and Ewing's sarcoma (ES), all accounting for about $75 \%$ of the cases [35•]. The cellular origin for these malignancies has been subject of intense investigation. In general, the resulting tumor phenotype is dictated by a combination of various factors, namely: the type of mutations accrued, the differentiation stage of the cell acquiring those mutations and the influence of the surrounding microenvironment $[35 \bullet, 36]$. For instance, specific mutations in undifferentiated MSC are responsible for the generation of ES. In contrast, mutations acquired in more specific subpopulations at different stages of differentiation (e.g., committed pre-osteoblasts) have been associated with the initiation of OS [35•].

Ewing's sarcoma is a primary bone and soft tissue tumor of mesenchymal origin. It is the second most frequent bone tumor, present almost exclusively in children and young adults. Its prognosis is directly correlated with its stage of detection, with a 5-year survival of 60-70\% [37]. The molecular pathogenesis of ES is defined by a recurrent chromosomal translocation between the EWSR1 gene and one of the five ETS transcription factors, which leads to the formation of a chimeric fusion gene [38, 39]. At least $80 \%$ of the fusion is presented as the EWS/FLI1 oncoprotein. Mechanistically, this resulting aberrant transcription factor appears to promote abnormal cellular growth by transcriptionally modulating a complex network of target genes during differentiation, including $m-F N G$, PDGF-C ligand and TGF $\beta$ RII [40], as well as disturbing RNA splicing and the ability of specific proteins to form functional complexes [41]. Historically, ES was thought to have originated from the endothelium (original proposition by James Ewing in 1921 and revisited in [42]). Later, a neuroectoderm origin was proposed, given that its 
constitutive cells express neural markers and are capable of neural differentiation [43]. Recently, a more mechanistic theory has been suggested, in which not only a mesenchymal origin proposed, but also the disrupting signaling capacity of the aberrant transcriptional factor/oncoprotein in MSC is related to tumor development [44]. In that sense, EWS/FLI1 is capable of inhibiting differentiation of mesenchymal precursors, such as adipogenic and osteogenic from murine MSC [45], and myogenic from C2C12 myoblasts [46]. These in vitro results were complemented with reports of an in vivo transformation of BM-derived MSC capable of generating tumors in immunocompromised mice with features similar to ES. These features include a small cell size, expression of ES-identifying markers, dependence on insulin-like growth factor 1 (IGF-1), and induction or repression of various EWS/FLI1 target genes [38]. In parallel, the expression of ES markers such as CD99 has been detected in MSC derived from ES patients [47]. Altogether, these data point toward the MSC as the permissive cell of origin for ES, which after transformation by the aberrant oncogene gives rise to this poorly differentiated mesenchymal tumor. Interestingly, this mesenchymal theory can be reconciled with the vascular origin of ES cells as initially proposed by Ewing. First, it has been shown that EWS/FLI1 modulate a number of angiogenic genes; second, the native FLI1 gene is pivotal during the molecular regulation of blood vessel formation; and third, a clear vascular mimicry has been identified in vivo within ES tumors, where their derived cells show the capacity of forming vascular-like structures [48]. In conclusion, ES cells may derive from MSC, which in turn exhibit mesenchymal and vascular potentials.

Additional information supporting the role of MSC as initiators of ES comes from their direct relationship with cancer stem cells (CSC) within the tumor. Suvà et al. isolated a subpopulation of tumor cells from ES biopsies, based on the conserved CSC marker CD133. The resulting $\mathrm{CD} 133^{\mathrm{Pos}}$ population retained the MSC plasticity and showed the ability to initiate and maintain tumor growth through serial transplantation in immunodeficiency mice, in sharp contrast with their $\mathrm{CD} 133^{\mathrm{Neg}}$ counterparts. In addition to re-establish the parental tumor phenotype, the percentage of $\mathrm{CD} 133^{\text {Pos }}$ cells in all subsequent tumors remained unaltered when compared with the original tumor, showing self-renewal capacity [49]. Collectively, these observations were consistent with CSC behavior. In another study, Riggi et al. demonstrated that the fusion gene/aberrant transcription factor EWS/ FLI1 is responsible for "reprogramming" MSC into a phenotype compatible with ES CSC [50]. They reported the expression of the embryonic stem cell genes Sox2, Oct4 and NANOG in MSC derived from pediatric samples, a phenomenon not evidenced in their adult counterparts. Mechanistically, EWS/FLI1 represses the promoter activity of miRNA145 on both itself and its in turn repressive activity over Sox2, demonstrating the effect of a single oncogene on reprogramming MSC into CSC.

Osteosarcoma is the most frequent tumor in bone, prevalent in adolescent and the elderly, which can be presented in a number of pathological variants depending on the stage of commitment of the osteoblastic cell [51]. The role of MSC as initiators of OS is less evident than with ES. In fact, more committed osteoprogenitors rather than undifferentiated MSC constitute the cell origin of OS. For instance, Rubio et al. reported that the excision of $\mathrm{p} 53 / \mathrm{Rb}$ in $\mathrm{BM}-\mathrm{MSC}$ generated leiomyosarcomas, while a similar deletion in further differentiated osteogenic cells generated OS-like tumors. Furthermore, they showed that the osteogenic differentiation stage of BM-derived MSC, not of cells derived from adipose tissue irrespective of their osteogenic stage, imposes the phenotype of in vivo OS development [52•]. On the other hand, Shimizu et al. showed that BM-MSC from Ink4a/Arf KO mice displayed at least two different subpopulations with different OS formation capacities: a highly tumorigenic population with bi-potent (osteochondral) differentiation potential, and a low tumorigenic population with tri-potent (not restricted) potential. Interestingly, when adipogenic differentiation was enforced in the two populations, the OS formation potential was significantly impaired. The authors concluded that the chondrogenic and adipogenic intrinsic programs may suppress the OS potential of MSC [53]. Collectively, and unlike in ES where undifferentiated MSC act as the cell of origin, more committed osteoprogenitors constitute the cell source of OS. Furthermore, the closer to an undifferentiated MSC phenotype, the less likely an OS will be generated [54].

In addition to the aforementioned link of MSC (and their direct cellular descendants) with primary neoplasms in the bone, their key perivascular residence and vascular support functions enable them to also participate in distant tumor formation.

3) Bone marrow-derived MSC in distant primary tumor formation

Neovascularization is required for tumor growth [55]. The TME exhibits features of a site with chronic inflammatory conditions. This local inflammation, along with intratumoral hypoxia and the activity of recruited cells such as the tumor-associated macrophages (TAMs) and a myriad of pro-angiogenic molecules, drives the formation of new blood vessels [2, 56, 57]. However, constant formation, regression and remodeling of vascular structures dominate inside the tumor, generating unstable, leaky, tortuous, dilated and immature vessels $[2,14,55]$. The underlying imbalance between anti- and pro-angiogenic factors is accompanied by pericyte coverage deficiencies, which in conjunction contribute to the typical vascular abnormalities seen in tumoral vasculature [13, 
58]. These deficiencies in pericytes include variable recruitment and coverage of the vasculature, dissociated pericyteECs physical interactions, and the secretion of an altered basement membrane $[14,59]$.

A number of reports have established that in response to tumor-derived molecular signals including inflammatory and hypoxic mediators, BM-derived progenitor cells of mesenchymal origin (BM-MSC) are recruited to the tumor stroma as tumor-associated fibroblasts (TAFs) [4-6, 16]. TAFs exhibit a myofibroblast phenotype and localize in both perivascular and parenchymal sites, contributing to the fibrovascular network expansion and tumor progression $[60-62,63 \cdot, 64 \bullet]$. In addition, they have a particular secretory capacity associated with tumor formation, growth and distant dissemination potential, thus contributing to the ultimate clinical outcome $[65,66]$. In that regard, they secrete factors associated with promoting cell growth, ECM remodeling, angiogenesis and tumor aggression, making them distinguishable from regular tissue resident fibroblasts [67]. Within the tumor fibrovascular network, two distinct populations of TAFs have been identified, recruited sequentially and based on the steps of tumor development. On the one hand, a pericytic population recruited from neighboring adipose tissue was involved in the constant neovascularization of the growing tumor. One the other hand, a fibroblastic population recruited from the distant BM related with ECM remodeling and tumor growth directly [68•].

In addition to MSC, the BM provides other cell sources that travel far to the tumor stroma, recruited by specific queues. For instance, VEGF-responsive BM-derived endothelial and hematopoietic precursors are necessary and sufficient to guarantee tumor angiogenesis $[3,69]$. On the other hand, mobilization and recruitment of BM-derived PDGFR $\beta+$ pericyte progenitor cells have also been described in tumors, presumably recruited under the influence of PDGF- $\beta$ ligand $[18,70]$. In conclusion, BM-derived cells are critical for tumor formation and growth, and subsequent invasive and metastatic potential.

\section{4) Pericytes in cancer dissemination}

Growth rate, invasion and metastasis in solid tumors constitute independent pathological traits regulated by different genetic mechanisms [71, 72]. Nevertheless, they share a common feature, the dependence on the TME and specifically the vasculature, as it provides access to oxygen and nutrients and serves as a dissemination route for malignant cells [7]. The metastatic process requires cancer cells to experience a series of steps, all potentially compromising their survival. These steps include escaping from the solid tumor to the vasculature (i.e., intravasation), traveling through the circulation and finding a suitable soil to disseminate and continue growing after migrating out from the local blood vessels (i.e., extravasation) [73].

Targeting both EC and pericytes has proven to be more effective at reducing tumor growth than EC alone [10]. However, the microvascular structure, and specifically vessel coating pericytes, seems to differentially affect primary tumor growth and dissemination potential [13]. In fact, poor pericyte coverage of tumor vessels has been correlated with poor patient prognosis [74], due to increased metastatic dissemination, as shown in colorectal and prostate cancer $[75,76]$. Various animal models have been used to demonstrate that the pericytes in the vasculature of primary tumors limit cancer cell metastasis. For instance, Xian et al. showed that in a genetic model of pericyte detachment from the vasculature $\left(\mathrm{PFGFB}^{\text {ret/ret }}\right.$ mice), insulinoma cancer cells were more prone to disseminate to liver, kidney and intestine compared with control mice [11]. In addition, Cooke et al. reported that the deficient pericyte coverage of tumor vasculature is correlated with an enhanced metastatic potential of breast cancer cells [12]. On the other hand, the TME composition, and specifically the presence of recruited BM-MSC, seems to directly affect the dissemination potential of cancer cells [7]. For example, co-transplanting MSC with weakly metastatic breast cancer cells into an orthotopic site increases their dissemination potential to the lungs [4]. This pro-metastatic effect is reversible and shortlived, and is mediated by the paracrine activity of the MSC-derived CCL5/Rantes, which enhances the motility and invasiveness of CCR5-expressing cancer cells. Similarly, co-injected MSC with OS cell lines enhance their lung invasion, through CXCR4 activation and VEGF expression [77, 78]. In addition to this signaling mechanisms, a bi-directional metabolic reprogramming between MSC and OS cells has been reported to explain the OS increased invasiveness. The OS cells induce an oxidative stress state in MSC, stimulating them to produce lactate. The secreted lactate then induces mitochondrial metabolism in cancer cells, favoring their high metabolic demand and promoting motility [79•].

Pericytes recruitment and attachment to the forming vessel is regulated mainly by the endothelial cell secretion of PDGF$\mathrm{B}$, which binds to its receptor (PDGFR- $\beta$ ), expressed by pericyte progenitors $[8,9]$. A positively charged retention motif in the C-terminus part of PDGF-B interacts with negatively charged disaccharide building blocks of heparan sulfate proteoglycans (HSPGs) in the pericellular matrix surrounding endothelial cells. This interaction generates the retention of PDGF-B surrounding the endothelial cell, thus creating a chemoattracting gradient for PDGFR- $\beta$-expressing pericytic progenitors close to the endothelium [80]. Modifications of these HSPGs by the secretion of heparanases and sulfatases 
have been associated with vascular structural changes in primary tumors and with a propensity to dissemination in various cancers $[81,82]$.

5) MSC at the target organ: the special case of the BM microenvironment

Skeletal metastases are common complications in various malignancies including melanoma, breast, prostate and kidney cancer, severely undermining the quality of life of patients and negatively impacting the overall prognosis. Despite the advances in knowledge about skeletal metastasis pathophysiology, most of the work has focused on the invasive capability of the circulating osteotropic cancer cell, ignoring the potential contribution of the target organ microenvironment [7]. Stromal elements within the BM microenvironment recently started to have acceptance as determinants for the invasion of osteotropic cancer cells. For instance, blocking PDGF-B signaling with the multi-target tyrosine kinase inhibitor Sunitinib impairs bone invasion of circulating osteotropic lung cancer cell lines [83]. Some of the anti-invasive effects of Sunitinib have been associated with structural changes in the BM vasculature, including the disruption of the perivascular niche through an increased cell death of BM stromal cells (i.e., BM-MSC). Despite this anti-metastatic effect, Sunitinib has shown to generate hematologic toxicity in patients, posing some restrictions on its clinical use [84].

Various lines of evidence have suggested that cellular elements within the BM may have a role during the formation of skeletal metastasis. First, during extravasation, the endothelium gets retracted upon interaction with the invading cancer cells, involving the Sdf-1/CXCL12 - CXCR4 molecular axis

Correa D.

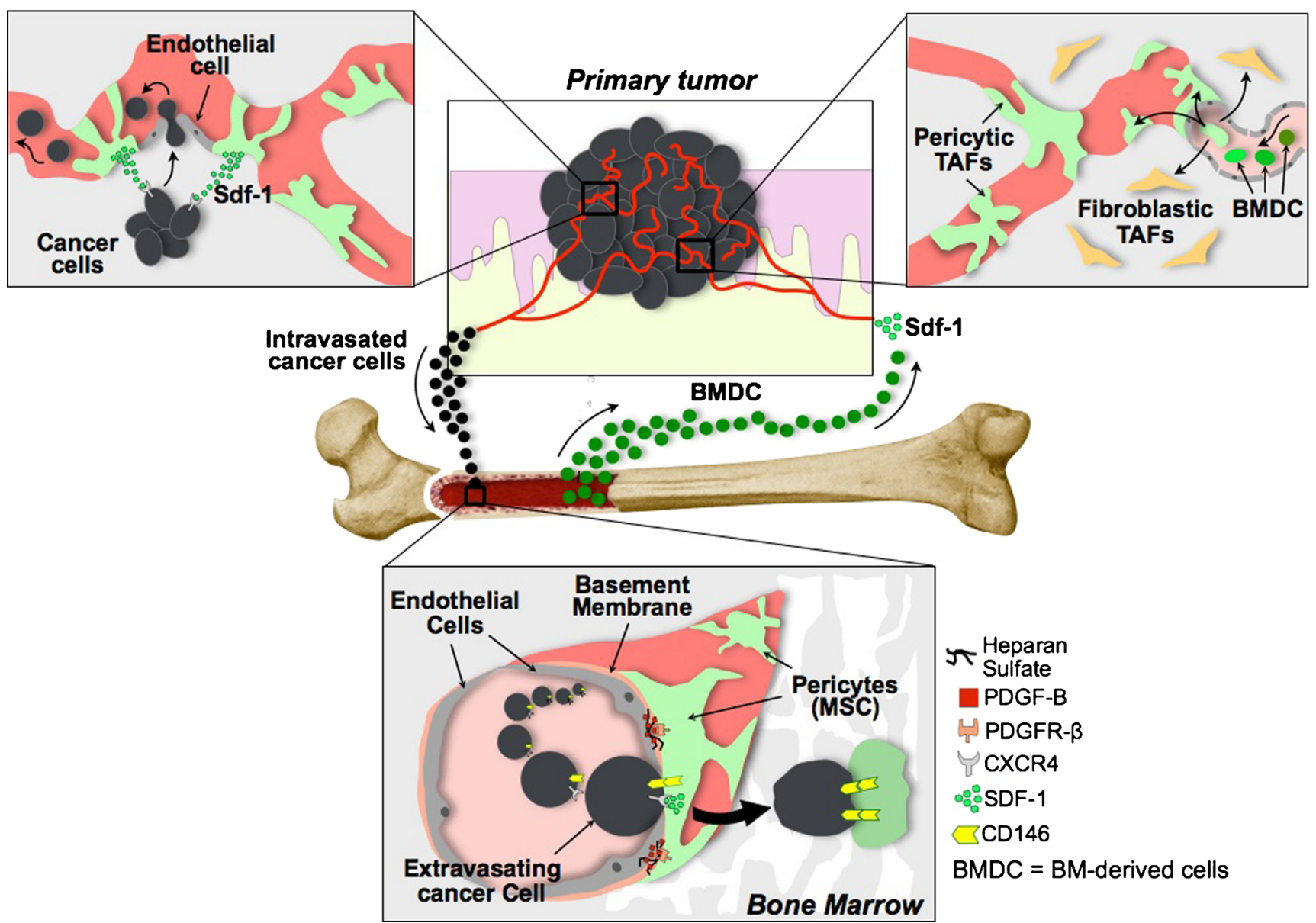

Fig. 1 Participation of MSC as pericytes in various aspects of cancer biology: BM-derived cells of mesenchymal origin (green cells) are recruited to the primary tumor following tumor-secreted signals (e.g., Sdf-1). At the primary tumor, they establish as tumor-associated fibroblasts (TAFs) both in the perivascular space (Pericytic TSFs) and the parenchyma (Fibroblastic TAFs). Later, cancer cells intravasate guided by pericyte-secreted signals (e.g., Sdf-1) and other cell types (e.g., macrophages, not shown here for clarity). Intravasated cancer cells find the target tissue (e.g., BM and liver), where they again follow an Sdf-1 gradient, and interact with MSC as pericytes via CD146. The multicellular complex then invades the tissue parenchyma 
[85]. In the BM, a subpopulation of perivascular cells, the CXCL12 Abundant Reticular (CAR) cells, constitute a rich source of Sdf-1/CXCL1 [30, 86•]. Second, various groups have reported the requirement of a functional pericyterecruiting PDGFR signaling and the presence of Sdf-1/ CXCL12-expressing BM-MSC for an adequate invasion of lung and breast cancer cells into bone [83, 87]. Third, invading cells physically interact with BM stromal cells, and this association affects various biological activities as well as the ultimate fate of cancer cells [7, 88]. All these data pointed to an active participation of stromal components within the BM during cancer cells invasion. However, the precise identity of those stromal components, their relationship with the blood vessels and the location where those physical cellular interactions occur during extravasation were still not fully clear. Uncoupling the vascular components (i.e.: disrupting pericyte-EC interactions) provided information to begin answering those questions. Correa et al. reported that in a genetic model of perturbed pericyte coverage of the vasculature (PDGF-B $^{\text {ret/ret }}$ mice), both BM and liver were selectively "protected" from invasion by intra-arterially injected B16F10 melanoma cells [89•]. They showed that as pericytes, BM-MSC are required for an efficient extravasation of melanoma cells. BM-MSC attract (via Sdf-1/CXCL12) and physically interact with (through co-expressed CD146) melanoma cells at the abluminal/perivascular space, initiating the transendothelial migration process. Both molecular axes have been linked with metastatic behavior and poor prognosis in patients with melanoma [90,91], becoming novel therapeutic targets [92]. This report also underscores the participation of BM-MSC in their perivascular localization as a cellular component of the hematopoietic stem cell niche, during the extravasation of circulating cancer cells into hematopoietic tissues such as the BM and the liver. In that respect, the stem cell niche in the BM has been previously established as a preferred target location for the initial invasion of osteotropic cancer cells [93].

Collectively, vascular components of the target tissue stroma, and specifically BM-MSC as pericytes, participate in the dissemination of circulating cancer cells into the BM. Moreover, their localization in special multi-cellular structures such as the stem cell niche in active hematopoietic tissues provides a new perspective not only in stem cell biology, but also in the analysis of cancer cells engraftment into the homing tissue. Acting as pericytes, BM-MSC unprecedentedly become central orchestrators during cancer invasion, by participating in chemoattraction, adhesion and migration of circulating osteotropic cancer cells into the BM parenchyma. Furthermore, some of the known mechanisms associated with invasion now can be linked to MSC, based on the accumulating literature supporting the expression of some of the participating molecules (e.g., CD146, Sdf-1/CXCL12) by those perivascular cells.

\section{Conclusion}

The TME is gaining acceptance as a critical component of tissues during the formation of primary and metastatic tumors. Cellular and structural components of the TME, especially those related to the vasculature, are now linked to key aspects of the tumor biology. With the recent identification of the MSC niche in a perivascular location (as pericytes) and the reports of the direct involvement of BM-MSC in cancer, these adult stem cells and their signaling molecules become recognized as central parts of the TME, thus constituting novel therapeutic targets. In addition, all this information provides evidence for a more comprehensive appreciation of MSC, once perceived exclusively as cellular progenitors.

In addition, to serve as the cellular origin of some bone malignancies (e.g., ES), the strategic localization of MSC as pericytes endows them with active/dynamic roles in cancer biology. These roles are exerted at various steps: 1) the formation of the tumor stroma as they are recruited from the BM to be part of the developing TME; 2) control of the intravasation of cancer cells into the tumor vasculature; 3 ) regulation of the extravasation of disseminating cancer cells once they find the target organ to initiate a distant metastatic tumor (Fig. 1). Importantly, the effect of the pericyte disruption during intravasation at the primary tumor does not reflect the situation during extravasation at the distant target organ. In the former, the defective pericyte coverage promotes distant dissemination [11], while in the latter, it selectively prevents invasion from circulating cancer cells [89•]. Understanding this differential effect can drive the design of novel therapeutic schemes in which coadjuvant interventions are added at the time of removal of the primary tumor to prevent new and to treat existing skeletal metastasis.

Finally, by exploiting their key perivascular location, BM-MSC may play additional roles in other aspects of the biology of skeletal metastasis, such as the quiescence/ dormancy of engrafted cancer cells, the angiogenic switch that triggers tumor growth and the local anti-tumor immune response.

Acknowledgments Drs. Caplan and Bonfield wish to thank Dr. Baisakhi Raychaurdhuri for their kind assistance in the reviewing of this article.

\section{Compliance with Ethical Standards}

Conflict of Interest Diego Correa reports a patent pending for Bone Marrow Pericytes/MSC: Gatekeepers Controlling Skeletal Metastasis from Melanoma; and a patent pending for In vivo platform for the identification of controlling elements during the process of metastatic cancer dissemination to target organs.

Human and Animal Rights and Informed Consent This article does not contain any studies with human or animal subjects performed by any of the authors. 


\section{References}

Papers of particular interest, published recently, have been highlighted as:

- Of importance

1. Heron M. Deaths: leading causes for 2011. Natl Vital Stat Rep. 2015;64:1-96.

2. Balkwill FR, Capasso M, Hagemann T. The tumor microenvironment at a glance. J Cell Sci. The Company of Biologists Ltd; 2012; 125: 5591-6.

3. Lyden D, Hattori K, Dias S, et al. Impaired recruitment of bone-marrow-derived endothelial and hematopoietic precursor cells blocks tumor angiogenesis and growth. Nat Med. 2001;7: 1194-201.

4. Karnoub AE, Dash AB, Vo AP, et al. Mesenchymal stem cells within tumour stroma promote breast cancer metastasis. Nature. 2007;449:557-63.

5. Mishra PJ, Merlino G. A traitor in our midst: mesenchymal stem cells contribute to tumor progression and metastasis. Future Oncol (London, England). 2008;4:745-9.

6. Spaeth E, Klopp A, Dembinski J, et al. Inflammation and tumor microenvironments: defining the migratory itinerary of mesenchymal stem cells. Gene Ther. 2008;15:730-8.

7. Joyce JA, Pollard JW. Microenvironmental regulation of metastasis. Nat Rev Cancer. 2009;9:239-52.

8. Armulik A, Abramsson A, Betsholtz C. Endothelial/pericyte interactions. Circ Res. 2005;97:512-23.

9. Gaengel K, Genove G, Armulik A, et al. Endothelial-mural cell signaling in vascular development and angiogenesis. Arterioscler Thromb Vasc Biol. 2009;29:630-8.

10. Bergers G, Song S, Meyer-Morse N, et al. Benefits of targeting both pericytes and endothelial cells in the tumor vasculature with kinase inhibitors. J Clin Invest. 2003;111:1287-95.

11. Xian X, Håkansson J, Ståhlberg A, et al. Pericytes limit tumor cell metastasis. J Clin Invest. 2006;116:642-51.

12. Cooke VG, LeBleu VS, Keskin D, et al. Pericyte depletion results in hypoxia-associated epithelial-to-mesenchymal transition and metastasis mediated by met signaling pathway. Cancer Cell. 2012;21: 66-81.

13. Gerhardt H, Semb H. Pericytes: gatekeepers in tumour cell metastasis? J Mol Med. 2008;86:135-44.

14. Gutiérrez R, Madrid JF, Varela H, et al. Pericytes. Morphofunction, interactions and pathology in a quiescent and activated mesenchymal cell niche. 2009; 24: 909-69.

15. Bergwerff M, Verberne ME, DeRuiter MC, et al. Neural crest cell contribution to the developing circulatory system: implications for vascular morphology? Circ Res. 1998;82:221-31.

16. Lamagna $\mathrm{C}$, Bergers $\mathrm{G}$. The bone marrow constitutes a reservoir of pericyte progenitors. J Leukoc Biol. 2006;80:677-81.

17. Hall AP. Review of the pericyte during angiogenesis and its role in cancer and diabetic retinopathy. Toxicol Pathol. 2006;34:763-75.

18. Rajantie I, Ilmonen M, Alminaite A, et al. Adult bone marrowderived cells recruited during angiogenesis comprise precursors for periendothelial vascular mural cells. Blood. 2004;104:2084-6.

19. Bergers G, Song S. The role of pericytes in blood-vessel formation and maintenance. Neuro-Oncology. 2005;7:452-64.

20. Diaz-Flores L, Gutierrez R, Gonzalez P, et al. Inducible perivascular cells contribute to the neochondrogenesis in grafted perichondrium. Anat Rec. 1991;229:1-8.
21. Díaz-Flores L, Gutiérrez R, Lopez-Alonso A, et al. Pericytes as a supplementary source of osteoblasts in periosteal osteogenesis. Clin Orthop Relat Res. 1992;275:280-6.

22. Brighton CT, Lorich DG, Kupcha R, et al. The pericyte as a possible osteoblast progenitor cell. Clin Orthop Relat Res. 1992;275:287-99.

23. Seldes R, Luchetti W. Similarities in the phenotypic expression of pericytes and bone cells. Clin Orthop Relat Res. 1998;346:95-103.

24. Traktuev DO, Merfeld-Clauss S, Li J, et al. A population of multipotent CD34-positive adipose stromal cells share pericyte and mesenchymal surface markers, reside in a periendothelial location, and stabilize endothelial networks. Circ Res. 2008;102:77-85.

25. Crisan M, Yap S, Casteilla L, et al. A perivascular origin for mesenchymal stem cells in multiple human organs. Cell Stem Cell. 2008;3:301-13.

26. Covas DT, Panepucci RA, Fontes AM, et al. Multipotent mesenchymal stromal cells obtained from diverse human tissues share functional properties and gene-expression profile with CD146+ perivascular cells and fibroblasts. Exp Hematol. 2008;36:642-54.

27. da Silva Meirelles L, Caplan AI, Nardi NB. In search of the in vivo identity of mesenchymal stem cells. Stem Cells. 2008;26:2287-99.

28. da Silva Meirelles L, Chagastelles PC, Nardi NB. Mesenchymal stem cells reside in virtually all post-natal organs and tissues. J Cell Sci. 2006;119:2204-13.

29. Khan WS, Tew SR, Adesida AB, et al. Human infrapatellar fat padderived stem cells express the pericyte marker $3 \mathrm{G} 5$ and show enhanced chondrogenesis after expansion in fibroblast growth factor2. Arthritis Res Ther. 2008;10(4):R74.

30. Sacchetti B, Funari A, Michienzi S, et al. Self-renewing osteoprogenitors in bone marrow sinusoids can organize a hematopoietic microenvironment. Cell. 2007;131:324-36.

31. Maes C, Kobayashi T, Selig MK, et al. Osteoblast precursors, but not mature osteoblasts, move into developing and fractured bones along with invading blood vessels. Dev Cell. 2010;19:329-44.

32. Bianco P, Riminucci M, Gronthos $\mathrm{S}$, et al. Bone marrow stromal stem cells: nature, biology, and potential applications. Stem Cells. 2001;19:180-92.

33. Caplan AI. All MSCs are pericytes? Cell Stem Cell. 2008;3: 229-30.

34. Brooks JSJ, Lee S. Contemporary diagnostics: sarcoma pathology update. Kraybill WG, Pollock RE, editors. Journal of Surgical Oncology. 2015; 111: 513-9.

35. Mutsaers AJ, Walkley CR. Cells of origin in osteosarcoma: mesenchymal stem cells or osteoblast committed cells? Bone. 2014;62: 56-63. A comprehensive review of the cellular origin of osteosarcoma, contrasting the theories of MSC vs committed osteoblasts.

36. Xiao W, Mohseny AB, Hogendoorn PCW, et al. Mesenchymal stem cell transformation and sarcoma genesis. Clin Sarcoma Res. 2013;3:10.

37. Gaspar N, Gaspar N, Hawkins DS, et al. Ewing Sarcoma: Current Management and Future Approaches Through Collaboration. J Clin Oncol Am Soc Clin Oncol. 2015;33:3036-46.

38. Castillero-Trejo Y, Eliazer S, Xiang L, et al. Expression of the EWS/ FLI-1 oncogene in murine primary bone-derived cells Results in EWS/FLI-1-dependent, ewing sarcoma-like tumors. Cancer Res. 2005;65:8698-705.

39. Potikyan G, France KA, Carlson MRJ, et al. Genetically defined EWS/FLI1 model system suggests mesenchymal origin of Ewing's family tumors. Lab Investig. 2008;88:1291-302.

40. Arvand A, Denny CT. Biology of EWS/ETS fusions in Ewing's family tumors. Oncogene. 2001;20:5747-54.

41. Janknecht R. EWS-ETS oncoproteins: the linchpins of Ewing tumors. Gene. 2005;363:1-14.

42. Ewing J. Diffuse endothelioma of bone. CA Cancer J Clin. 1972;22:95-8. 
43. Cavazzana AO, Magnani JL, Ross RA, et al. Ewing's sarcoma is an undifferentiated neuroectodermal tumor. Prog Clin Biol Res. 1988;271:487-98.

44. Tirode F, Laud-Duval K, Prieur A, et al. Mesenchymal stem cell features of ewing tumors. Cancer Cell. 2007;11:421-9.

45. Torchia EC, Jaishankar S, Baker SJ. Ewing tumor fusion proteins block the differentiation of pluripotent marrow stromal cells. Cancer Res. 2003;63:3464-8.

46. Eliazer S, Spencer J, Ye D, et al. Alteration of mesodermal cell differentiation by EWS/FLI-1, the oncogene implicated in Ewing's sarcoma. Mol Cell Biol. 2003;23:482-92.

47. Amaral AT, Manara MC, Berghuis D, et al. Characterization of human mesenchymal stem cells from ewing sarcoma patients. Pathogenetic implications. PLoS ONE. 2014;9:85814-4.

48. van der Schaft DWJ, Hillen F, Pauwels P, et al. Tumor cell plasticity in Ewing sarcoma, an alternative circulatory system stimulated by hypoxia. Cancer Res. 2005;65:11520-8.

49. Suva ML, Riggi N, Stehle JC, et al. Identification of cancer stem cells in Ewing's sarcoma. Cancer Res. 2009;69:1776-81.

50. Riggi N, Suva ML, De Vito C, et al. EWS-FLI-1 modulates miRNA145 and SOX2 expression to initiate mesenchymal stem cell reprogramming toward Ewing sarcoma cancer stem cells. Genes Dev. 2010;24:916-32.

51. Mirabello L, Troisi RJ, Savage SA. Osteosarcoma incidence and survival rates from 1973 to 2004. Cancer. 2009;115:1531-43.

52. Rubio R, Gutierrez-Aranda I, Sáez-Castillo AI, et al. The differentiation stage of p53-Rb-deficient bone marrow mesenchymal stem cells imposes the phenotype of in vivo sarcoma development. Oncogene. 2012;32:4970-80. Key paper demonstrating that the stage of osteogenic differentiation determines the ultimate phenotype of the developing sarcoma. It also shows that the origin for osteosarcoma is the BM-MSC-derived osteogenic progenitor and not the undifferentiated BM-MSC.

53. Shimizu T, Ishikawa T, Sugihara E. c-MYC overexpression with loss of Ink4a/Arf transforms bone marrow stromal cells into osteosarcoma accompanied by loss of adipogenesis. Oncogene. 2010;29: 5687-99.

54. Lin PP, Pandey MK, Jin F, et al. Targeted mutation of p53 and Rb in mesenchymal cells of the limb bud produces sarcomas in mice. Carcinogenesis. 2009;30:1789-95.

55. Carmeliet P, Jain RK. Molecular mechanisms and clinical applications of angiogenesis. Nature. 2011;473:298-307.

56. Grivennikov SI, Greten FR, Karin M. Immunity, inflammation, and cancer. Cell. 2010;140:883-99.

57. Hanahan D, Weinberg RA. Hallmarks of cancer: the next generation. Cell. 2011;144:646-74.

58. Gerhardt H, Betsholtz C. Endothelial-pericyte interactions in angiogenesis. Cell Tissue Res. 2003;314:15-23.

59. Abramsson A, Berlin O, Papayan H, et al. Analysis of mural cell recruitment to tumor vessels. Circulation. 2002;105:112-7.

60. Mishra PJ, Mishra PJ, Humeniuk R, et al. Carcinoma-associated fibroblast-like differentiation of human mesenchymal stem cells. Cancer Res. 2008;68:4331-9.

61. Spaeth EL, Dembinski JL, Sasser AK, et al. Mesenchymal stem cell transition to tumor-associated fibroblasts contributes to fibrovascular network expansion and tumor progression. PLoS ONE. 2009;4, e4992.

62. Kidd S, Spaeth E, Dembinski JL, et al. Direct evidence of mesenchymal stem cell tropism for tumor and wounding microenvironments using in vivo bioluminescent imaging. Stem Cells. 2009;27: 2614-23.

63. Lin P, Correa D, Kean TJ, et al. Serial transplantation and long-term engraftment of intra-arterially delivered clonally derived mesenchymal stem cells to injured bone marrow. Mol Ther. 2014;22:160-8. Key paper showing the journey of systemically-injected MSC, recognizing an injury site (mouse model of bone marrow injury), where they engraft repopulating their natural habitat, the perivascular space.

64. Dembinski JL, Wilson SM, Spaeth EL, et al. Tumor stroma engraftment of gene-modified mesenchymal stem cells as anti-tumor therapy against ovarian cancer. Cytotherapy. 2013;15:20-2. This paper confirms the tumor tropism of MSC after systemic injection, where they differentiate into perivascular myofibroblasts. As a result, IFN- $\beta$ expressing MSC significantly impacted the growth of murine ovarian carcinomas.

65. Yazhou C, Wenlv S, Weidong Z, et al. Clinicopathological significance of stromal myofibroblasts in invasive ductal carcinoma of the breast. Tumour Biol. 2004;25:290-5.

66. Tsujino T, Seshimo I, Yamamoto H, et al. Stromal myofibroblasts predict disease recurrence for colorectal cancer. Clin Cancer Res. 2007;13:2082-90

67. Silzle T, Randolph GJ, Kreutz M, et al. The fibroblast: sentinel cell and local immune modulator in tumor tissue. Int $\mathrm{J}$ Cancer. 2004; 108:173-80.

68. Kidd S, Spaeth E, Watson K, et al. Origins of the tumor microenvironment: quantitative assessment of adipose-derived and bone marrow-derived stroma. PLoS ONE. 2012;7: e30563. This is a key paper where the authors show that the bone marrow and the surrounding adipose tissue are indeed the sources for distinct populations of tumor associated fibroblasts (TAF).

69. LaRue AC, Masuya M, Ebihara Y, et al. Hematopoietic origins of fibroblasts: I. In vivo studies of fibroblasts associated with solid tumors. Exp Hematol. 2006;34:208-18.

70. Song S, Ewald AJ, Stallcup W, et al. PDGFRbeta + perivascular progenitor cells in tumours regulate pericyte differentiation and vascular survival. Nat Cell Biol. 2005;7:870-9.

71. Nguyen DX, Massagué J. Genetic determinants of cancer metastasis. Nat Rev Genet. 2007;8:341-52.

72. Nguyen DX, Bos PD, Massagué J. Metastasis: from dissemination to organ-specific colonization. Nat Rev Cancer. 2009;9:274-84.

73. Chambers AF, Groom AC, MacDonald IC. Dissemination and growth of cancer cells in metastatic sites. Nat Rev Cancer. 2002;2:563-72.

74. O'Keeffe MB, Devlin AH, Burns AJ, et al. Investigation of pericytes, hypoxia, and vascularity in bladder tumors: association with clinical outcomes. Oncol Res. 2008;17:93-101.

75. Yonenaga Y, Mori A, Onodera H, et al. Absence of smooth muscle actin-positive pericyte coverage of tumor vessels correlates with hematogenous metastasis and prognosis of colorectal cancer patients. Oncology. 2005;69:159-66.

76. Welén K, Jennbacken K, Tesan T, et al. Pericyte coverage decreases invasion of tumour cells into blood vessels in prostate cancer xenografts. Prostate Cancer Prostatic Dis. 2009;12:41-6.

77. Fontanella R, Pelagalli A, Nardelli A, et al. A novel antagonist of CXCR4 prevents bone marrow-derived mesenchymal stem cellmediated osteosarcoma and hepatocellular carcinoma cell migration and invasion. Cancer Lett. 2016;370:100-7.

78. Zhang P, Dong L, Long H, et al. Homologous mesenchymal stem cells promote the emergence and growth of pulmonary metastases of the rat osteosarcoma cell line UMR-106. Oncol Lett. 2014;8: $127-32$.

79. Bonuccelli G, Avnet S, Grisendi G, et al. Role of mesenchymal stem cells in osteosarcoma and metabolic reprogramming of tumor cells. Oncotarget. 2014;5:7575-88. This paper elegantly shows the tight bidirectional crosstalk between MSC and osteosarcoma cells in vitro, where a mutual metabolic reprogramming of them increases the cancer cells migratory ability.

80. Lindblom P, Gerhardt H, Liebner S, et al. Endothelial PDGF-B retention is required for proper investment of pericytes in the microvessel wall. Genes Dev. 2003;17:1835-40. 
81. Goldshmidt O, Zcharia E, Abramovitch R, et al. Cell surface expression and secretion of heparanase markedly promote tumor angiogenesis and metastasis. Proc Natl Acad Sci U S A. 2002;99: 10031-6.

82. Yang Y, MacLeod V, Bendre M, et al. Heparanase promotes the spontaneous metastasis of myeloma cells to bone. Blood. 2005;105: 1303-9.

83. Catena R, Luis-Ravelo D, Antón I, et al. PDGFR signaling blockade in marrow stroma impairs lung cancer bone metastasis. Cancer Res. 2011;71:164-74.

84. Gkountouvas A, Kostoglou-Athanassiou I, Veniou E, Repousis P, et al. Hematologic toxicity in patients treated with sunitinib for advanced thyroid cancer. Thyroid. 2010;20:597-600.

85. Lee B-C, Lee T-H, Avraham S, et al. Involvement of the chemokine receptor CXCR4 and its ligand stromal cell-derived factor 1alpha in breast cancer cell migration through human brain microvascular endothelial cells. Mol Cancer Res. 2004;2:327-38.

86. Mendelson A, Frenette PS. Hematopoietic stem cell niche maintenance during homeostasis and regeneration. Nat Med. 2014;20:833-46. This is a key paper where an in depth study of the bone marrow stem cell niche is provided. The different phenotypes of MSC as pericytes and molecular regulators are discussed.

87. Corcoran KE, Trzaska KA, Fernandes H, et al. Mesenchymal stem cells in early entry of breast cancer into bone marrow. PLoS ONE. 2008;3:e2563.
88. Yoneda T, Hiraga T. Crosstalk between cancer cells and bone microenvironment in bone metastasis. Biochem Biophys Res Commun. 2005;328:679-87.

89. Correa D, Somoza RA, Lin P, et al. Mesenchymal stem cells regulate melanoma cancer cells extravasation to bone and liver at their perivascular niche. Int J Cancer. 2016;138(2):417-27. This is a key report that establishes the required presence of MSC/pericytes in hematopoietic tissues (bone marrow and liver) for an efficient melanoma cancer cell invasion into their target organs. For the first time, it establishes the distinct role of pericytes in intravasation and extravasation of cancer cells.

90. Lehmann JM, Riethmüller G, Johnson JP. MUC18, a marker of tumor progression in human melanoma, shows sequence similarity to the neural cell adhesion molecules of the immunoglobulin superfamily. Proc Natl Acad Sci U S A. 1989;86:9891-5.

91. Scala S, Ottaiano A, Ascierto PA, et al. Expression of CXCR4 predicts poor prognosis in patients with malignant melanoma. Clin Cancer Res. 2005;11:1835-41.

92. McGary EC, Heimberger A, Mills L, et al. A fully human antimelanoma cellular adhesion molecule/MUC18 antibody inhibits spontaneous pulmonary metastasis of osteosarcoma cells in vivo. Clin Cancer Res. 2003;9:6560-6.

93. Shiozawa Y, Pedersen EA, Havens AM, et al. Human prostate cancer metastases target the hematopoietic stem cell niche to establish footholds in mouse bone marrow. J Clin Invest. 2011;121: 1298-312. 